\title{
Support-Limited Generalized Uncertainty Relations on Fractional Fourier Transform
}

\author{
Xiaotong Wang1, Guanlei $\mathrm{Xu}^{\mathbf{2}^{*}}$ \\ ${ }^{1}$ Navgation Department of Dalian Naval Academy, Dalian, China \\ ${ }^{2}$ Ocean Department of Dalian Naval Academy, Dalian, China \\ Email: ${ }^{*} x g \mid$ 86@163.com
}

Received 16 June 2015; accepted 18 August 2015; published 21 August 2015

Copyright (C) 2015 by authors and Scientific Research Publishing Inc.

This work is licensed under the Creative Commons Attribution International License (CC BY). http://creativecommons.org/licenses/by/4.0/

cC) (i) Open Access

\begin{abstract}
This paper investigates the generalized uncertainty principles of fractional Fourier transform (FRFT) for concentrated data in limited supports. The continuous and discrete generalized uncertainty relations, whose bounds are related to FRFT parameters and signal lengths, were derived in theory. These uncertainty principles disclose that the data in FRFT domains may have much higher concentration than that in traditional time-frequency domains, which will enrich the ensemble of generalized uncertainty principles.
\end{abstract}

\section{Keywords}

Discrete Fractional Fourier Transform (DFRFT), Uncertainty Principle, Frequency-Limiting Operator

\section{Introduction}

In information processing, the uncertainty principle plays an important role in elementary fields, and data concentration is often considered carefully via the uncertainty principle [1]-[8]. In continuous signals, the supports are assumed to be infinite, based on which various uncertainty relations [1] [2] [9]-[21] [22] have been presented. However, in practice, both the supports of time and frequency are often limited for N-point discrete signals. In such case, the infinite support fails to hold true. In limited supports, some papers such as [23]-[26] have discussed the uncertainty principle in conventional time-frequency domains for continuous and discrete cases and some conclusions are achieved that can be taken as our special cases in the following sections. However, none of them has covered the FRFT in terms of Heisenberg uncertainty principles that have been widely used in various

${ }^{*}$ Corresponding author. 
fields [4]-[6]. Therefore, there has a great need to discuss the uncertainty relations in FRFT domains. As the rotation of the traditional FT [27], FRFT [5] [6] [28]-[30] has some special properties with its transform parameter and sometimes yields the better results such as the detection of LFM signal [31]. Readers can see more details on FRFT in [6] and [32] and so on.

In this paper, we extend the Heisenberg uncertainty principle in FRFT domain for both discrete and continuous cases for the $\varepsilon$-concentrated signals or the signals with finite supports. It is shown that these bounds are connected with lengths of the supports and FRFT parameters. In a word, there have been no reported papers covering these results and conclusions, and most of them are new or novel.

\section{Preliminaries}

\subsection{Definition of DFRFT}

Here, we first briefly review the definition of FRFT. For given continuous signal $x(t) \in L^{1}(R) \cap L^{2}(R)$ and $\|x(t)\|_{2}=1$, its FRFT [6] is defined as

$$
\begin{aligned}
X_{\alpha}(u) & =F_{\alpha}(x(t))=\int_{-\infty}^{\infty} x(t) K_{\alpha}(u, t) \mathrm{d} t \\
& = \begin{cases}\sqrt{\frac{(1-i \cot \alpha)}{2 \pi}} \cdot \mathrm{e}^{\frac{i u^{2} \cot \alpha}{2}} \int_{-\infty}^{\infty} \mathrm{e}^{\frac{-i u t}{\sin \alpha}} \mathrm{e}^{\frac{i t^{2} \cot \alpha}{2}} x(t) \mathrm{d} t & \alpha \neq n \pi \\
x(t) & \alpha=2 n \pi \\
x(-t) & \alpha=(2 n \pm 1) \pi\end{cases}
\end{aligned}
$$

where $n \in \mathrm{Z}$ and $i$ is the complex unit, $\alpha$ is the transform parameter defined as that in [6]. In addition, $F_{\alpha} F_{\beta}(x(t))=F_{\alpha+\beta}(x(t))$. If $\alpha=-\beta, F_{\alpha} F_{\beta}(x(t))=x(t)$, i.e., the inverse FRFT

$x(t)=\int_{-\infty}^{\infty} X_{\alpha}(u) K_{-\alpha}(u, t) \mathrm{d} u$.

However, unlike the discrete FT, there are a few definitions for the DFRFT [32], but not only one. In this paper, we will employ the definition defined as follows [6] [32]:

$$
\hat{x}(k)=\sum_{n=1}^{N} \sqrt{(1-i \cot \alpha) / N} \cdot \mathrm{e}^{\frac{i k^{2} \cot \alpha}{2}} \mathrm{e}^{\frac{-i k n}{N \sin \alpha}} \mathrm{e}^{\frac{i n^{2} \cot \alpha}{2 N^{2}}} \tilde{x}(n)=\sum_{n=1}^{N} u_{\alpha}(k, n) \cdot \tilde{x}(n), 1 \leq n, k \leq N .
$$

Clearly, if $\alpha=\pi / 2$, (2) reduces to the traditional discrete FT [6] [32]. Also, we can rewrite definition (2) as

$$
\hat{X}_{\alpha}=U_{\alpha} \tilde{X}
$$

where $U_{\alpha}=\left[u_{\alpha}(k, n)\right]_{N \times N}, \quad \tilde{X}=[\tilde{x}(n)]_{N \times 1}$.

For DFRFT, we have the following property [5] [6] [32]:

$$
\left\|\hat{X}_{\alpha}\right\|_{2}=\left\|U_{\alpha} \tilde{X}\right\|_{2}=1 \text {. }
$$

More details on DFRFT can be found in [6] and [32].

\subsection{Frequency-Limiting Operators}

Definition 1: Let $x(t)$ be a complex-valued signal with $\|x(t)\|_{L^{2}(R)}=1$ and its FRFT $X_{\alpha}(u)$, if there is a function $G_{\alpha}(u)$ vanishing outside $W_{\alpha}\left(W_{\alpha}\right.$ is a measurable set) such that $\left\|X_{\alpha}(u)-G_{\alpha}(u)\right\|_{L^{2}(R)} \leq \varepsilon_{\alpha}\left(\varepsilon_{\alpha}\right.$ is a small value with $\left.0<\varepsilon_{\alpha}<1\right)$, then $X_{\alpha}(u)$ is $\varepsilon_{\alpha}$-concentrated.

Specially, if $\alpha=0$, then definition 1 reduces to the case in time domain [23] [24]. If $\alpha=\pi / 2$, then definition 1 reduces to the case in traditional frequency domain [23] [24]. The $\varepsilon_{\alpha}$ can be calculated after the $W_{\alpha}$ is 
fixed because $G_{\alpha}(u) \subseteq X_{\alpha}(u)$ and $\left\|X_{\alpha}(u)\right\|_{L^{2}(R)}=\|x(t)\|_{L^{2}(R)}=1$. Therefore, $\left\|X_{\alpha}(u)-G_{\alpha}(u)\right\|_{L^{2}(R)} \leq \varepsilon_{\alpha}$

Definition 2: Generalized frequency-limiting operator $P_{W_{\alpha}}$ is defined as

$$
\left(P_{W_{\alpha}} x\right)(t) \equiv \int_{W_{\alpha}} X_{\alpha}(u) K_{-\alpha}(u, t) \mathrm{d} u, X_{\alpha}(u)=F_{\alpha}(x(t)) .
$$

If $\alpha=0$, then definition 2 is the time-limiting operator [23] [24]. If $\alpha=\pi / 2$, then definition 2 is the traditional frequency-limiting operator [23] [24]. Definitions 1 and 2 disclose the relation between $\varepsilon_{\alpha}$ and $W_{\alpha}$. For the discrete case, we have the following definitions.

Definition 3: Let $\tilde{x}(n) \in l^{2}(R) \quad\left(n=1, \cdots, N\right.$ with) be a discrete sequence with $\|\tilde{x}(n)\|_{l^{2}(R)}=1$ and its DFRFT $\hat{x}(k)$, if there is a sequence $\hat{x}_{\alpha}^{\prime}(k)$ satisfying $\left\|\hat{x}_{\alpha}^{\prime}(k)\right\|_{0}=N_{\alpha} \quad\left(N_{\alpha} \leq N\right)$ such that $\left\|\hat{x}_{\alpha}(k)-\hat{x}_{\alpha}^{\prime}(k)\right\|_{l^{2}(R)} \leq \varepsilon_{\alpha} \quad\left(\varepsilon_{\alpha}\right.$ is a small value with $\left.0<\varepsilon_{\alpha}<1\right)$, then $\hat{x}(k)$ is $\varepsilon_{\alpha}$-concentrated.

Here, \|\|$_{0}$ is the 0 -norm operator that counts the non-zero elements.

Definition 4: Generalized discrete frequency-limiting operator $P_{N_{\alpha}}$ is defined as

$\left(P_{N_{\alpha}} \tilde{x}\right)(n)=\sum_{k=1}^{N} \chi_{N \alpha} \hat{x}(k) u_{-\alpha}(k, n)$ with $\hat{x}(k)$ is the DFRFT of $\tilde{x}(n)$ and $\chi_{N_{\alpha}}$ is the character function on $N_{\alpha}\left(N_{\alpha} \leq N\right)$.

Clearly, definitions 3 and 4 are the discrete extensions of definitions 1 and 2. They have the similar physical meaning. These definitions are introduced for the first time, the traditional cases [23] [24] are only their special cases. Definition 3 and 4 disclose the relation between $\varepsilon_{\alpha}$ and $N_{\alpha}$.

\subsection{The Continuous Heisenberg Uncertainty Principles}

As shown in introduction, the existed continuous generalized uncertainty relations [9]-[21] are mainly for the infinite supports. Here, we discuss the case of finite support. First we introduce the following lemma.

Lemma 1: $\left\|P_{W_{\alpha}} P_{W_{\beta}}\right\|_{H S}=\sqrt{\frac{\left|W_{\alpha}\right|\left|W_{\beta}\right|}{|\sin (\alpha-\beta)|}}$, where $\|\cdot\|_{H S}$ denotes the Frobenius norm operator.

Proof: From the definition of the operator $P_{W_{\alpha}} P_{W_{\beta}}$ in definition 2, we have

$$
\left(P_{W_{\alpha}} P_{W_{\beta}} x\right)(t)=\int_{W_{\alpha}} K_{-\alpha}(v, t)\left(\int_{-\infty}^{\infty} K_{\alpha}(v, t)\left(\int_{W_{\beta}} K_{-\beta}(t, u) X_{\beta}(u) \mathrm{d} u\right) \mathrm{d} t\right) \mathrm{d} v .
$$

Exchange the locations of the integral operators, we obtain

$$
\left(P_{W_{\alpha}} P_{W_{\beta}} x\right)(t)=\int_{W_{\alpha}} K_{-\alpha}(v, t)\left(\int_{W_{\beta}} K_{\alpha-\beta}(v, u) X_{\beta}(u) \mathrm{d} u\right) \mathrm{d} v,
$$

so that

$$
\left(P_{W_{\alpha}} P_{W_{\beta}} X\right)(t)=\int_{W_{\beta}}\left(\int_{W_{\alpha}} K_{-\alpha}(v, t) K_{\alpha-\beta}(v, u) \mathrm{d} v\right) X_{\beta}(u) \mathrm{d} u .
$$

Set $q(u, t)=\left\{\begin{array}{ll}\int_{W_{\alpha}} K_{-\alpha}(v, t) K_{\alpha-\beta}(v, u) \mathrm{d} v, & u \in W_{\beta} \\ 0, & \text { else }\end{array}\right.$, we have

$$
\left(P_{W_{\alpha}} P_{W_{\beta}} x\right)(t)=\int_{W_{\beta}} q(u, t) X_{\beta}(u) \mathrm{d} u .
$$

Now, we know that [see the proof of (3.1) in 25]

$$
\left\|P_{W_{\alpha}} P_{W_{0}}\right\|_{H S}^{2}=\int_{W_{\beta}} \int_{-\infty}^{\infty}|q(u, t)|^{2} \mathrm{~d} u \mathrm{~d} t .
$$


Let $g_{u}(t)=q(u, t)$, then

$$
\begin{aligned}
F_{\alpha}\left(g_{u}(t)\right) & =\int_{-\infty}^{\infty} K_{\alpha}(u, t)\left(\int_{W_{\alpha}} K_{-\alpha}(v, t) K_{\alpha-\beta}(v, u) \mathrm{d} v\right) \mathrm{d} t \\
& =\int_{W_{\alpha}} K_{\alpha-\beta}(v, u)\left(\int_{-\infty}^{\infty} K_{-\alpha}(v, t) K_{\alpha}(u, t) \mathrm{d} t\right) \mathrm{d} v \\
& =\int_{W_{\alpha}} K_{\alpha-\beta}(v, u) \delta(u-v) \mathrm{d} v=\chi_{W_{\alpha}} \cdot K_{\alpha-\beta}(v, u),
\end{aligned}
$$

where $\chi_{W_{\alpha}}$ is the character function of the set $W_{\alpha}$. Therefore, via Parseval's theorem [6] and the definition of FRFT in (1) we have

$$
\int_{-\infty}^{\infty}\left|g_{u}(t)\right|^{2} \mathrm{~d} t=\int_{-\infty}^{\infty}\left|F_{\alpha}\left(g_{u}(t)\right)\right|^{2} \mathrm{~d} u=\int_{-\infty}^{\infty}\left|\chi_{W_{\alpha}} \cdot K_{\alpha-\beta}(v, u)\right|^{2} \mathrm{~d} u=\frac{\left|W_{\alpha}\right|}{|\sin (\alpha-\beta)|} .
$$

Hence, we obtain the final result

$$
\left\|P_{W_{\alpha}} P_{W_{\beta}}\right\|_{H S}^{2}=\int_{W_{\beta}} \int_{-\infty}^{\infty}|q(u, t)|^{2} \mathrm{~d} t \mathrm{~d} u=\int_{W_{\beta}} \frac{\left|W_{\alpha}\right|}{|\sin (\alpha-\beta)|} \mathrm{d} u=\frac{\left|W_{\alpha}\right|\left|W_{\beta}\right|}{|\sin (\alpha-\beta)|} .
$$

Now we give the first theorem.

Theorem 1: Let $W_{\alpha}\left(W_{\beta}\right)$ be a measurable set and suppose $X_{\alpha}(u)\left(X_{\beta}(u)\right)$ is the FRFT of $x(t)$ for transform parameter $\alpha(\beta)$, such that $X_{\alpha}(u)\left(X_{\beta}(u)\right)$ is $\varepsilon_{W_{\alpha}}\left(\varepsilon_{W_{\beta}}\right)$-concentrated on $W_{\alpha}\left(W_{\beta}\right)$. Then

$$
\left|W_{\alpha}\right|\left|W_{\beta}\right| \geq\left(1-\varepsilon_{W_{\alpha}}-\varepsilon_{W_{\beta}}\right)^{2}|\sin (\alpha-\beta)| .
$$

Proof: Since $\left\|P_{W_{\alpha}}\right\|_{L^{2}(R)}=\sup _{f(t) \in L^{2}(R)} \frac{\left\|P_{W_{\alpha}} x(t)\right\|_{L^{2}(R)}}{\|x(t)\|_{L^{2}(R)}}$, therefore we can find such $x(t)$ that makes $\left\|P_{W_{\alpha}}\right\|_{L^{2}(R)}=1$.

Meanwhile, via triangle inequality and the definitions of concentration we have

$$
\begin{aligned}
\left\|x(t)-P_{W_{\alpha}} P_{W_{\beta}} x(t)\right\|_{L^{2}(R)} & =\left\|x(t)-P_{W_{\alpha}} x(t)+P_{W_{\alpha}}\left(x(t)-P_{W_{\beta}} x(t)\right)\right\|_{L^{2}(R)} \\
& \leq\left\|x(t)-P_{W_{\alpha}} x(t)\right\|_{L^{2}(R)}+\left\|P_{W_{\alpha}}\left(x(t)-P_{W_{\beta}} x(t)\right)\right\|_{L^{2}(R)} \leq \varepsilon_{W_{\alpha}}+\varepsilon_{W_{\beta}} .
\end{aligned}
$$

At the same time, we know $\left\|x(t)-P_{W_{\alpha}} P_{W_{\beta}} x(t)\right\|_{L^{2}(R)} \geq\|x(t)\|_{L^{2}(R)}-\left\|P_{W_{\alpha}} P_{W_{\beta}} x(t)\right\|_{L^{2}(R)}=1-\left\|P_{W_{\alpha}} P_{W_{\beta}} x(t)\right\|_{L^{2}(R)}$, so that

$$
\left\|P_{W_{\alpha}} P_{W_{\beta}} x(t)\right\|_{L^{2}(R)} \geq 1-\left(\varepsilon_{W_{\beta}}+\varepsilon_{W_{\alpha}}\right),
$$

i.e., $\frac{\left\|P_{W_{\alpha}} P_{W_{\beta}} x(t)\right\|_{L^{2}(R)}}{\|x(t)\|_{L^{2}(R)}} \geq 1-\left(\varepsilon_{W_{\beta}}+\varepsilon_{W_{\alpha}}\right)$.

Therefore, $\left\|P_{W_{\alpha}} P_{W_{\beta}}\right\|_{L^{2}(R)} \geq \frac{\left\|P_{W_{\alpha}} P_{W_{\beta}} x(t)\right\|_{L^{2}(R)}}{\|x(t)\|_{L^{2}(R)}} \geq 1-\left(\varepsilon_{W_{\beta}}+\varepsilon_{W_{\alpha}}\right)$.

From [24] [27], we know that $\left\|P_{W_{\alpha}} P_{W_{\beta}}\right\|_{H S} \geq\left\|P_{W_{\alpha}} P_{W_{\beta}}\right\|_{L^{2}(R)}$.

Use the above two results, we obtain 


$$
\left\|P_{W_{\alpha}} P_{W_{\beta}}\right\|_{H S}=\sqrt{\frac{\left|W_{\alpha}\right|\left|W_{\beta}\right|}{|\sin (\alpha-\beta)|}} \geq\left\|P_{W_{\alpha}} P_{W_{\beta}}\right\|_{L^{2}(R)},
$$

i.e., $\sqrt{\left|W_{\alpha}\right|\left|W_{\beta}\right|} \geq\left\|P_{W_{\alpha}} P_{W_{\beta}}\right\|_{L^{2}(R)} \sqrt{|\sin (\alpha-\beta)|}$.

Hence, $\left|W_{\alpha}\right|\left|W_{\beta}\right| \geq\left\|P_{W_{\alpha}} P_{W_{\beta}}\right\|_{L^{2}(R)}^{2}|\sin (\alpha-\beta)| \geq\left(1-\varepsilon_{W_{\alpha}}-\varepsilon_{W_{\beta}}\right)^{2}|\sin (\alpha-\beta)|$. The special case $\alpha-\beta=n \pi$ is trivial. Here, we find that when $\alpha-\beta=\pi / 2+k \pi$, (4) reduce to the traditional case in Theorem 2 [(3.1), 25].

Obviously, this bound is different from that [20] of infinite case. In [20], the main involved objects are the variances of the signal in infinite supports. Here the measurable sets $\left(W_{\alpha}, W_{\beta}\right)$ are involved, which is instructive for the discrete case in the next section. If $\varepsilon_{W_{\alpha}}=\varepsilon_{W_{\beta}}=0$, what will happen? Clearly, it is impossible. From the conclusion [33], if $\varepsilon_{W_{\alpha}}=0$, then $\varepsilon_{W_{\beta}} \neq 0$, otherwise $W_{\beta}=\infty$, which is in conflict with that $W_{\beta}$ is measurable and limited. Therefore, in the continuous case, $\varepsilon_{W_{\alpha}}=\varepsilon_{W_{\beta}}=0$ cannot hold true. However, what about the discrete case? The next section will answer.

\section{The Discrete Heisenberg Uncertainty Principles}

\subsection{The Uncertainty Relation}

First let us introduce a lemma.

Lemma 3: $\left\|P_{N_{\alpha}} P_{N_{\beta}}\right\|_{F}=\sqrt{\frac{N_{\alpha} \cdot N_{\beta}}{N|\sin (\alpha-\beta)|}}$, where \|\|$_{F}$ is the Frobenius matrix norm.

Proof: From the definition of the operator $P_{N_{\alpha}} P_{N_{\beta}}$ in definition 4, we have

$$
\left(P_{N_{\alpha}} P_{N_{\beta}} \tilde{x}\right)(n)=\sum_{k=1}^{N} \chi_{N_{\alpha}} u_{\alpha}(k, n) \sum_{v=1}^{N} \chi_{N_{\beta}} \hat{x}(v) u_{-\beta}(k, v) .
$$

Exchange the locations of the sum operators, we obtain

$$
\begin{aligned}
\left(P_{N_{\alpha}} P_{N_{\beta}} \tilde{x}\right)(n) & =\sum_{v=1}^{N} \sum_{k=1}^{N} \chi_{N_{\alpha}} \chi_{N_{\beta}} u_{\alpha}(k, n) \hat{x}(v) u_{-\beta}(k, v) \\
& =\sum_{k=1}^{N} \chi_{N_{\alpha}} \sum_{v=1}^{N} \chi_{N_{\beta}} \hat{x}(v) u_{\alpha-\beta}(n, v) .
\end{aligned}
$$

Hence, according to the definition of the Frobenius matrix norm [27] [34] and the definition of DFRFT, we have

$$
\left\|P_{N_{\alpha}} P_{N_{\beta}}\right\|_{F}=\left(\sum_{k=1}^{N} \chi_{N_{\alpha}} \sum_{v=1}^{N} \chi_{N_{\beta}}\left|u_{\alpha-\beta}(n, v)\right|^{2}\right)^{1 / 2}=\sqrt{\frac{N_{\alpha} \cdot N_{\beta}}{N|\sin (\alpha-\beta)|}} .
$$

In the similar manner with the continuous case, we can obtain $\frac{\left\|P_{N_{\alpha}} P_{N_{\beta}} \tilde{x}(n)\right\|_{I^{2}(R)}}{\|\tilde{x}(n)\|_{l^{2}(R)}} \geq 1-\left(\varepsilon_{\alpha}+\varepsilon_{\beta}\right)$. Since $\left\|P_{N_{\alpha}} P_{N_{\beta}}\right\|_{F} \geq\left\|P_{N_{\alpha}} P_{N_{\beta}}\right\|_{l^{2}(R)}=\sup _{x(n) \in l^{2}(R)} \frac{\left\|P_{N_{\alpha}} P_{N_{\beta}} \tilde{x}(n)\right\|_{l^{2}(R)}}{\|\tilde{x}(n)\|_{l^{2}(R)}}$, we have $\sqrt{\frac{N_{\alpha} \cdot N_{\beta}}{N|\sin (\alpha-\beta)|}}=\left\|P_{N_{\alpha}} P_{N_{\beta}}\right\|_{F} \geq \frac{\left\|P_{N_{\alpha}} P_{N_{\beta}} \tilde{x}(n)\right\|_{I^{2}(R)}}{\|\tilde{x}(n)\|_{l^{2}(R)}} \geq 1-\left(\varepsilon_{\alpha}+\varepsilon_{\beta}\right)$, thus, we get 
$N_{\alpha} \cdot N_{\beta} \geq N \cdot\left(1-\varepsilon_{\alpha}-\varepsilon_{\beta}\right)^{2}|\sin (\alpha-\beta)|$. Therefore, we can obtain the following theorem 2.

Theorem 2: Let $\hat{x}_{\alpha}(k)\left(\hat{x}_{\beta}(k)\right)$ be the DFRFT of the time sequence $\tilde{x}(n) \in l^{2}(R) \quad(n=1, \cdots, N)$ for transform parameter $\alpha(\beta)$, with $\hat{x}_{\alpha}(k)\left(\hat{x}_{\beta}(k)\right) \varepsilon_{\alpha}\left(\varepsilon_{\beta}\right)$-concentrated on index set $N \quad\left(\varepsilon_{\alpha} \varepsilon_{\beta} \neq 0\right)$. Let $N_{\alpha}\left(N_{\beta}\right)$ be the numbers of nonzero entries in $\hat{x}_{\alpha}^{\prime}(k)\left(\hat{x}_{\beta}^{\prime}(k)\right.$ respectively). Then

$$
\left\{\begin{array}{ll}
N_{\alpha} \cdot N_{\beta} \geq N \cdot\left(1-\varepsilon_{\alpha}-\varepsilon_{\beta}\right)^{2}|\sin (\alpha-\beta)|, & \alpha-\beta \neq n \pi \\
N_{\alpha} \cdot N_{\beta} \geq 1, & \alpha-\beta=n \pi
\end{array} .\right.
$$

Here, we find that when $\alpha-\beta=\pi / 2+k \pi$, (5) reduce to the traditional case in Theorem 3 [(3.9), 25].

\subsection{The Extensions}

Set $\varepsilon_{\alpha}=\varepsilon_{\beta}=0$ in theorem 2, we can obtain the following theorem 3 directly.

Theorem 3: Let $\hat{x}_{\alpha}(k)\left(\hat{x}_{\beta}(k)\right)$ be the DFRFT of the time sequence $\tilde{x}(n) \in l^{2}(R) \quad(n=1, \cdots, N)$ with length $N . N_{\alpha}\left(N_{\beta}\right)$ counts the numbers of nonzero entries in $\hat{x}_{\alpha}(k)\left(\hat{x}_{\beta}(k)\right.$ respectively). Then

$$
\left\{\begin{array}{ll}
N_{\alpha} \cdot N_{\beta} \geq N \cdot|\sin (\alpha-\beta)|, & \alpha-\beta \neq n \pi \\
N_{\alpha} \cdot N_{\beta} \geq 1, & \alpha-\beta=n \pi
\end{array} .\right.
$$

Clearly, theorem 3 is a special case of theorem 2. Also, this theorem can be derived via theorem 1 in [26]. Differently, we obtain this result in a different way. Here we note that since $\|\tilde{x}(n)\|_{I^{2}(R)}=1$, there is at least one non-zero element in every FRFT domain for $\alpha-\beta=n \pi$. Therefore, $N_{\alpha} \cdot N_{\beta} \geq 1$ for $\alpha-\beta=n \pi$.

Through setting special value for $\beta=0$ in theorem 3 , we have

\section{Corollary 1:}

$$
\left\{\begin{array}{ll}
N_{\alpha} \cdot N_{0} \geq N \cdot|\sin \alpha| & \alpha \neq n \pi \\
N_{\alpha} \cdot N_{0} \geq 1 & \alpha=n \pi
\end{array} .\right.
$$

Proof: Now we prove corollary 1 in the sense of sampling and mathematical solution for better understanding these relations. Without loss of generality, we often assume that the continuous signal $x(t)$ (the continuous version of $\tilde{x}(n))$ is band-limited, then $\tilde{x}(n)$ is obtained through sampling $x(t)$. From the sequence length $N$ in the definition of DFRFT in (2), we know the sampling period defined as $T_{s}: T_{s}=1 \quad\left(\tilde{x}(n)=\tilde{x}\left(n T_{s}\right)\right.$ implies this result). We assume there is no aliasing after sampling in the FRFT domain, then from the sampling Theorem, we know that all the energy of $\hat{x}_{\alpha}(k)$ are limited within the scope $\left[-\frac{N|\sin \alpha|}{2 T_{s}}, \frac{N|\sin \alpha|}{2 T_{s}}\right]$ [32] [35], i.e., all the energy of $\hat{x}_{\alpha}(k)$ must be within $[m+1, m+\lceil N|\sin \alpha|\rceil](0 \leq m, m+\lceil N|\sin \alpha|\rceil \leq N)$. Without loss of generality, we assume $m=0$ based on the shifting property of FRFT [6] [32], i.e., all the energy of $\hat{x}_{\alpha}(k)$ must be within $[1,[N|\sin \alpha|\rceil]$. Let $n_{1}, n_{2}, \cdots, n_{N_{t}}$ be the sites where $\tilde{x}(n)$ is nonzero, and $\tilde{x}\left(n_{l}\right)$ $\left(l=1, \cdots, N_{t}\right)$ be the corresponding nonzero elements of $\tilde{x}(n)$. Accordingly, from the definition of DFRFT [6] [32], we have

$$
\hat{x}_{\alpha}(k)=\sum_{l=1}^{N_{t}} \sqrt{(1-i \cot \alpha) / N} \cdot \mathrm{e}^{\frac{i k^{2} \cot \alpha}{2}} \mathrm{e}^{\frac{-i k n_{l}}{N \sin \alpha}} \mathrm{e}^{\frac{i n_{l}^{2} \cot \alpha}{2 N^{2}}} \tilde{x}\left(n_{l}\right), k=1,2, \cdots,\lceil N|\sin \alpha|\rceil \text { and } \tilde{x}\left(n_{l}\right) \neq 0 .
$$

We rewrite (8) in terms of matrices and vectors. Define the matrix $Z_{k, l} \equiv \sqrt{(1-i \cot \alpha) / N} \cdot D_{\alpha}\left(n_{l}, k\right)$, where $D_{\alpha}\left(n_{l}, k\right)=\mathrm{e}^{\frac{i k^{2} \cot \alpha}{2}} \mathrm{e}^{\frac{-i k n_{l}}{N \sin \alpha}} \mathrm{e}^{\frac{i n_{l}^{2} \cot \alpha}{2 N^{2}}}$, then we obtain 


$$
\underline{\hat{x}_{\alpha}}=Z b,
$$

where $\underline{\hat{x}_{\alpha}}=\left[\hat{x}_{\alpha}(1), \cdots, X_{\alpha}(\lceil N|\sin \alpha|\rceil)\right]^{\mathrm{T}}, \quad Z=\left(Z_{k, l}\right)_{\lceil N|\sin \alpha|\rceil \times N_{t}}$ and $b=\left[\tilde{x}\left(n_{1}\right), \cdots, \tilde{x}\left(n_{N_{t}}\right)\right]^{\mathrm{T}}$.

Clearly, $Z$ is a $\lceil N|\sin \alpha|\rceil \times N_{t}$ matrix, which includes $\frac{\lceil N|\sin \alpha|\rceil}{N_{0}}$ matrixes with dimensions of $N_{t} \times N_{t}$ so that we can rewrite matrix $Z$ as $Z=\left[Z_{1}, Z_{2}, \cdots, Z_{s}, \cdots\right]^{\mathrm{T}}$ and $\underline{\hat{x}_{\alpha}}=\left[\hat{x}_{\alpha, 1}, \hat{x}_{\alpha, 2}, \cdots, \hat{x}_{\alpha, s}, \cdots\right]^{\mathrm{T}}$, where $s=1,2, \cdots, \frac{\lceil N|\sin \alpha|\rceil}{N_{0}}$.

From the definition of DFRFT, we know that the bases $\sqrt{(1-i \cot \alpha) / N} \cdot \mathrm{e}^{\frac{i k^{2} \cot \alpha}{2}} \mathrm{e}^{\frac{-i k n_{l}}{N \sin \alpha}} \mathrm{e}^{\frac{i n_{l}^{2} \cot \alpha}{2 N^{2}}}$ (for different ks and $n_{l} s$ ) are mutually orthogonal [6] [32]. Therefore, the different rows are not correlated so that $Z_{s}$ $\left(s=1,2, \cdots, \frac{\lceil N|\sin \alpha|\rceil}{N_{0}}\right)$ is nonsingular and $\hat{x}_{\alpha, s}=Z_{s} b$ can be rewritten as $\left(Z_{s}\right)^{-1} \hat{x}_{\alpha, s}=b$. Since every element in $b$ is not zero and $Z_{s}$ is nonsingular, then there must be a non-zero element in $\hat{x}_{\alpha, s}$ at least. Other wise, $b=0$, which is in conflict with $b \neq 0$. Therefore, in every $\hat{x}_{\alpha, s}\left(s=1,2, \cdots, \frac{\lceil N|\sin \alpha|\rceil}{N_{0}}\right)$ there is at least one non-zero element. Therefore, there are at least $N_{\alpha} \geq \frac{\lceil N|\sin \alpha|\rceil}{N_{0}}$ non-zero elements in the DFRFT domain in total. Thus, theorem 3 is verified.

Furthermore, we can obtain the following more general uncertainty relation associated with DFRFT.

Clearly, if $|\sin \alpha|<1$ and $|\sin (\alpha-\beta)|<1$, then the generalized uncertainty bounds are lower than the traditional cases. Therefore, the generalized uncertainty principles show that the resolution will be higher.

Theorem 4: Let $\hat{x}_{\alpha_{l}}(k) \quad(l=1,2, \cdots, L)$ be the DFRFT of the time sequence $\tilde{x}(n) \in l^{2}(R) \quad(n=1, \cdots, N$ and $N>L$ ) with length $N$ and $\|\tilde{x}(n)\|_{l^{2}(R)}=1 . N_{\alpha_{l}}$ counts the number of nonzero elements in $\hat{x}_{\alpha_{l}}(k)$. Then

$$
\frac{N_{\alpha_{1}}+N_{\alpha_{2}}+\cdots+N_{\alpha_{L}}}{L} \geq \sqrt{N \cdot|\sin \xi|} \text {, where } \xi=\inf _{\substack{1 \leq l_{1}, l_{2} \leq L \\ l_{1} \neq l_{2}}}\left\{\left|\alpha_{l_{1}}-\alpha_{l_{2}}\right|\right\} \text {. }
$$

Proof: From the assumption and the definition of DFRFT [6] [32], we know

$$
\tilde{x}(n)=\sum_{k_{1}=1}^{N} u_{-\alpha_{1}}\left(n, k_{1}\right) \hat{x}_{\alpha_{1}}\left(k_{1}\right)=\sum_{k_{2}=1}^{N} u_{-\alpha_{2}}\left(n, k_{2}\right) \hat{x}_{\alpha_{2}}\left(k_{2}\right) \text { for } n=1,2, \cdots, N .
$$

where $u_{-\alpha_{l}}\left(n, k_{l}\right)=\sqrt{\left(1-i \cot \alpha_{l}\right) / N} \cdot \mathrm{e}^{\frac{-i k_{l}^{2} \cot \alpha_{l}}{2}} \mathrm{e}^{\frac{i k_{l} n}{N \sin \alpha_{l}}} \mathrm{e}^{\frac{-i n^{2} \cot \alpha_{l}}{2 N^{2}}},(l=1,2, \cdots, L)$.

Therefore, let $\tilde{X}=\left[\begin{array}{llll}\tilde{x}(1) & \tilde{x}(2) & \cdots & \tilde{x}(N)\end{array}\right]^{\mathrm{T}}$, we have [26]

$$
\tilde{X}^{\mathrm{T}} \tilde{X}=\left[\begin{array}{llllll}
\hat{x}_{\alpha_{l_{1}}}(1) & \hat{x}_{\alpha_{l_{1}}}(2) \cdots & \cdots & \hat{x}_{\alpha_{l_{1}}}(N)
\end{array}\right]\left[\begin{array}{c}
u_{-\alpha_{l_{1}}}^{\mathrm{T}}(1,:) \\
u_{-\alpha_{l_{1}}}^{\mathrm{T}}(2,:) \\
\vdots \\
u_{-\alpha_{l_{1}}}^{\mathrm{T}}(N,:)
\end{array}\right]\left[\begin{array}{llll}
u_{-\alpha_{l_{2}}}(1,:) & u_{-\alpha_{l_{2}}}(2,:) & \cdots & u_{-\alpha_{l_{2}}}(N,:)
\end{array}\right]\left[\begin{array}{c}
\hat{x}_{-\alpha_{l_{2}}}(1) \\
\hat{x}_{-\alpha_{l_{2}}}(2) \\
\vdots \\
\hat{x}_{-\alpha_{l_{2}}}(N)
\end{array}\right] \text {, }
$$

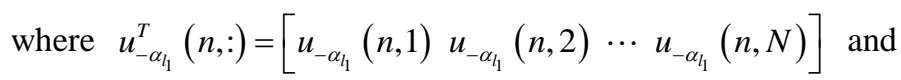


$u_{-\alpha_{l_{2}}}(n,:)=\left[u_{-\alpha_{l_{2}}}(n, 1) u_{-\alpha_{l_{2}}}(n, 2) \cdots u_{-\alpha_{l_{2}}}(n, N)\right]^{\mathrm{T}}$ with $n=1,2, \cdots, N$ and $l_{1}, l_{2}=1,2, \cdots, L$ with $l_{1} \neq l_{2}$.

Hence, we obtain

$$
\tilde{X}^{\mathrm{T}} \tilde{X}=\sum_{n=1}^{N} \sum_{k=1}^{N} \hat{x}_{\alpha_{l_{1}}}(n)\left\langle u_{-\alpha_{l_{1}}}(n,:), u_{-\alpha_{l_{2}}}(k,:)\right\rangle \hat{x}_{\alpha_{l_{2}}}(k) .
$$

Set $M_{\left(l_{1}, l_{2}\right)}=\sup _{n, k}\left(\left|\left\langle u_{-\alpha_{l_{1}}}(n,:), u_{-\alpha_{l_{2}}}(k,:)\right\rangle\right|\right)$, then

$$
\begin{aligned}
\tilde{X}^{\mathrm{T}} \tilde{X} & \leq \sum_{n=1}^{N} \sum_{k=1}^{N}\left|\hat{x}_{\alpha_{l_{1}}}(n)\left\langle u_{-\alpha_{l_{1}}}(n,:), u_{-\alpha_{l_{2}}}(k,:)\right\rangle \hat{x}_{\alpha_{l_{2}}}(k)\right| \\
& \leq \sum_{s_{1}=1}^{N_{\alpha_{l_{1}}}} \sum_{s_{2}=1}^{N_{\alpha_{l_{2}}}}\left|\hat{x}_{\alpha_{l_{1}}}\left(s_{1}\right)\right| \cdot M_{\left(l_{1}, l_{2}\right)} \cdot\left|\hat{x}_{\alpha_{l_{2}}}\left(s_{2}\right)\right| \\
& \leq M_{\left(l_{1}, l_{2}\right)} \sum_{s_{1}=1}^{N_{\alpha_{1}}} \sum_{s_{2}=1}^{N_{\alpha_{l_{2}}}}\left|\hat{x}_{\alpha_{l_{1}}}\left(s_{1}\right)\right| \cdot\left|\hat{x}_{\alpha_{l_{2}}}\left(s_{2}\right)\right|
\end{aligned}
$$

Using the triangle inequality, we have

$$
\begin{aligned}
\left|\hat{x}_{\alpha_{1}}\left(s_{1}\right)\right| \cdot\left|\hat{x}_{\alpha_{l_{2}}}\left(s_{2}\right)\right| \leq \frac{\left|\hat{x}_{\alpha_{l_{1}}}\left(s_{1}\right)\right|^{2}+\left|\hat{x}_{\alpha_{l_{2}}}\left(s_{2}\right)\right|^{2}}{2} \text {, hence } \\
\tilde{X}^{\mathrm{T}} \tilde{X} \leq M_{\left(l_{1}, l_{2}\right)} \sum_{s_{1}=1}^{N_{\alpha_{l_{1}}}} \sum_{s_{2}=1}^{N_{\alpha_{l_{2}}}} \frac{\left|\hat{x}_{\alpha_{l_{1}}}\left(s_{1}\right)\right|^{2}+\left|\hat{x}_{\alpha_{l_{2}}}\left(s_{2}\right)\right|^{2}}{2} \\
=M_{\left(l_{1}, l_{2}\right)} \cdot\left(\sum_{s_{1}=1}^{N_{\alpha_{l_{1}}}} \sum_{s_{2}=1}^{N_{\alpha_{l_{2}}}} \frac{\left|\hat{x}_{\alpha_{l_{1}}}\left(s_{1}\right)\right|^{2}}{2}+\sum_{s_{1}=1}^{N_{\alpha_{l_{1}}}} \sum_{s_{2}=1}^{N_{\alpha_{l_{2}}}} \frac{\left|\hat{x}_{\alpha_{l_{2}}}\left(s_{2}\right)\right|^{2}}{2}\right) \\
=M_{\left(l_{1}, l_{2}\right)} \cdot\left(\sum_{s_{2}=1}^{N_{\alpha_{l_{2}}}}\left(\sum_{s_{1}=1}^{N_{\alpha_{l_{1}}}} \frac{\left.\hat{x}_{\alpha_{l_{1}}}\left(s_{1}\right)\right|^{2}}{2}\right)+\sum_{s_{1}=1}^{N_{\alpha_{l_{1}}}}\left(\sum_{s_{2}=1}^{N_{\alpha_{l_{2}}}} \frac{\left|\hat{x}_{\alpha_{l_{2}}}\left(s_{2}\right)\right|^{2}}{2}\right) .\right.
\end{aligned}
$$

From $\|\tilde{x}(n)\|_{2}=1$ and Parseval's principle [6], we obtain

$$
\sum_{s_{1}=1}^{N_{\alpha_{h_{1}}}} \frac{\left|\hat{x}_{\alpha_{l_{1}}}\left(s_{1}\right)\right|^{2}}{2}=\sum_{s_{2}=1}^{N_{\alpha_{l_{2}}}} \frac{\left|\hat{x}_{\alpha_{l_{2}}}\left(s_{2}\right)\right|^{2}}{2}=\frac{1}{2} .
$$

Hence

$$
\tilde{X}^{\mathrm{T}} \tilde{X} \leq M_{\left(l_{1}, l_{2}\right)} \cdot\left(\sum_{s_{2}=1}^{N_{\alpha_{l_{2}}}} \frac{1}{2}+\sum_{s_{1}=1}^{N_{\alpha_{1}}} \frac{1}{2}\right)=M_{\left(l_{1}, l_{2}\right)} \cdot \frac{N_{\alpha_{l_{1}}}+N_{\alpha_{l_{2}}}}{2} .
$$

Therefore, we obtain

$$
\begin{aligned}
\tilde{X}^{\mathrm{T}} \tilde{X} & \leq M_{(1,2)} \cdot \frac{N_{1}+N_{2}}{2}, \\
\tilde{X}^{\mathrm{T}} \tilde{X} & \leq M_{(1,3)} \cdot \frac{N_{1}+N_{3}}{2}, \\
& \vdots \\
\tilde{X}^{\mathrm{T}} \tilde{X} & \leq M_{(L-1, L)} \cdot \frac{N_{L-1}+N_{L}}{2} .
\end{aligned}
$$


Adding all the above inequalities, we have

$$
\Gamma_{L}^{2} \cdot \tilde{X}^{\mathrm{T}} \tilde{X} \leq \sup _{\substack{1 \leq l_{1}, l_{2} \leq L \\ l_{1} \neq l_{2}}}\left\{M_{\left(l_{1}, l_{2}\right)}\right\} \frac{(L-1) \cdot\left(N_{1}+N_{2}+\cdots+N_{L}\right)}{2} \text { with } \Gamma_{L}^{2}=\frac{L \cdot(L-1)}{2 \times 1} .
$$

Similarly, from $\|\tilde{X}(n)\|_{2}=1$ and Parseval's principle [6], we obtain $\tilde{X}^{\mathrm{T}} \tilde{X}=1$, hence

$$
\frac{(L-1) \cdot\left(N_{1}+N_{2}+\cdots+N_{L}\right)}{2} \geq \frac{\Gamma_{L}^{2}}{\sup _{\substack{1 \leq 1_{1}, l_{1} \leq L \\ l_{1} \neq l_{2}}}\left\{M_{\left(1_{1}, l_{2}\right)}\right\}} .
$$

From the definition and property of DFRFT [6] [32] we have

$$
\sup _{\substack{1 \leq 1_{1}, l_{2} \leq L \\ l_{1} \neq l_{2}}}\left\{M_{\left(l_{1}, l_{2}\right)}\right\}=\sup _{\substack{1 \leq s_{1}, s_{2} \leq N \\ 1 \leq l_{1}, l_{2} \leq L, l_{1} \neq l_{2}}}\left(\left|K_{-\alpha_{l_{1}}+\alpha_{l_{2}}}\left(s_{1}, s_{2}\right)\right|\right)=\sup _{\substack{1 \leq 1_{1}, s_{2} \leq N \\ 1 \leq l_{1}, l_{2} \leq L, l_{1} \neq l_{2}}}\left(\left|\frac{1}{\sqrt{N \cdot \mid \sin \left(\alpha_{l_{1}}-\alpha_{l_{2}}\right)}}\right|\right)=\frac{1}{\sqrt{N \cdot|\sin \xi|}}
$$

with $\xi=\inf _{\substack{1 \leq l_{1}, l_{1} \leq L \\ l_{1} \neq l_{2}}}\left(\left|\alpha_{l_{1}}-\alpha_{l_{2}}\right|\right)$.

Hence, we finally obtain the proof

$$
\frac{N_{1}+N_{2}+\cdots+N_{L}}{L} \geq \sqrt{N \cdot|\sin \xi|} \text { with } \xi=\inf _{\substack{1 \leq l_{1}, l_{2} \leq L \\ l_{1} \neq l_{2}}}\left(\left|\alpha_{l_{1}}-\alpha_{l_{2}}\right|\right) .
$$

\section{The Simulation}

In this section we give an example to show that the data in FRFT domains may have much higher concentration than that in traditional time-frequency domains.

Now considering the chirp signal $f(n)$ with $n \in[1,1000] \mathrm{s}$ and sampling period $T_{s}=1 \mathrm{~s}$, $f(n)=\cos \left(0.001\left(n+3 n^{2}\right)\right)$ (see Figure 1(a)).

Clearly, we can obtain from Figure 1 that $N_{0}=1000, N_{\alpha=\pi / 2}=300, N_{\alpha=1.061 \pi / 2}=1$. Therefore, we have $N_{0} N_{\pi / 2} \approx 300,000>N_{0} N_{\alpha=1.061 \pi / 2}=1000$. This verifies that the data in FRFT domains may have much higher concentration than that in traditional time-frequency domains. (Note here that if the transformed coefficient is less than 0.1, then we take it as zero value. See Figure 1(b) and Figure 1(c)).

\section{Conclusion}

In practice, we often process the data with limited lengths for both the continuous ( $\varepsilon$-concentrated) and discrete signals. Especially for the discrete data, not only the supports are limited, but also they are sequences of data

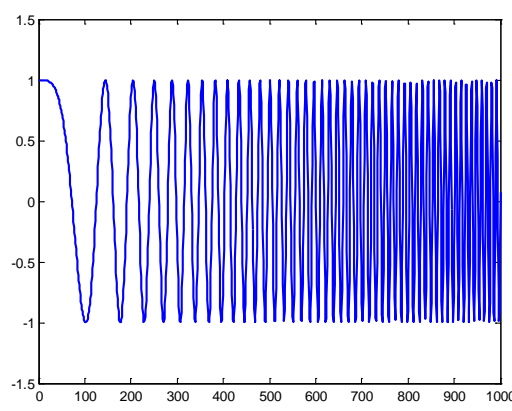

(a)

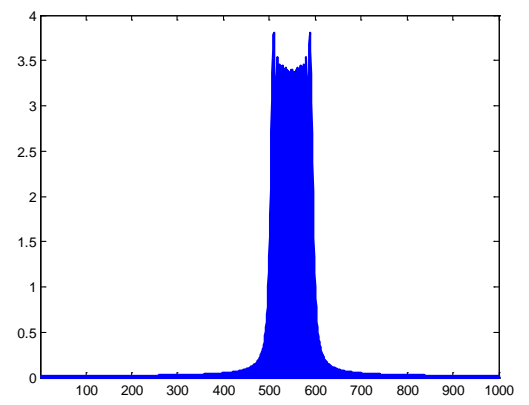

(b)

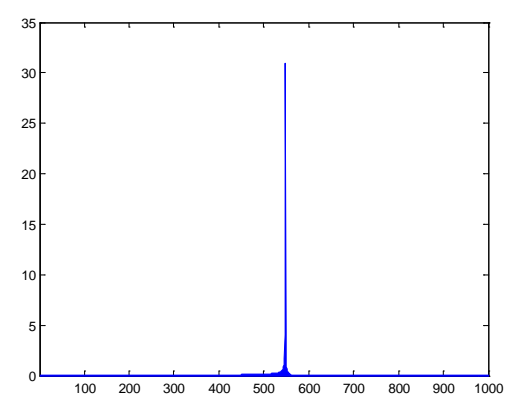

(c)

Figure 1. The simulation of a signal with its FRFT and FT. (a) The original signal in time domain; (b) The FT of the signal (i.e., the traditional frequency domain); (c) The FRFT of the signal (i.e., the FRFT domain). 
points whose number of non-zero elements is countable accurately. This paper discussed the generalized uncertainty relations on FRFT in term of data concentration. We show that the uncertainty bounds are related to the FRFT parameters and the support lengths. These uncertainty relations will enrich the ensemble of FRFT. Moreover, these uncertainty relations will help find the optimal filtering parameters [31] such as [6] [34] [36]. Our simulation also shows that the data in FRFT domains may have much higher concentration than that in traditional time-frequency domains.

\section{Acknowledgements}

We will thank Professor R. Tao very much for his valuable suggestions in improving our work. This work was fully supported by the NSFCs (61002052 and 61471412) and partly supported by the NSFC (61250006) and Third Term of 2110 in Dalian Navy Academy.

\section{References}

[1] Folland, G.B. and Sitaram, A. (1997) The Uncertainty Principle: A Mathematical Survey. The Journal of Fourier Analysis and Applications, 3, 207-238. http://dx.doi.org/10.1007/BF02649110

[2] Selig, K.K. (2001) Uncertainty Principles Revisited, Technische Universitat Munchen. Technical Report. http://www-lit.ma.tum.de/veroeff/quel/010.47001.pdf

[3] Loughlin, P.J. and Cohen, L. (2004) The Uncertainty Principle: Global, Local, or Both? IEEE Transactions on Signal Processing, 52, 1218-1227. http://dx.doi.org/10.1109/TSP.2004.826160

[4] Dembo, A., Cover, T.M. and Thomas, J.A. (2001) Information Theoretic Inequalities. IEEE Transactions on Information Theory, 37, 1501-1508. http://dx.doi.org/10.1109/18.104312

[5] Zhang, X.D. (2002) Modern Signal Processing. 2nd Edition, Tsinghua University Press, Beijing, 362.

[6] Tao, R., Qi, L. and Wang, Y. (2004) Theory and Applications of the Fractional Fourier Transform. Tsinghua University Press, Beijing.

[7] Maassen, H. (1988) A Discrete Entropic Uncertainty Relation, Quantum Probability and Applications. V, SpringerVerlag, New York, 263-266.

[8] Birula, I.B. (1985) Entropic Uncertainty Relations in Quantum Mechanics, Quantum Probability and Applications II, In: Accardi, L. and von Waldenfels, W., Eds., Lecture Notes in Mathematics 1136, Springer, Berlin, 90.

[9] Shinde, S. and Vikram, M.G. (2001) An Uncertainty Principle for Real Signals in the Fractional Fourier Transform Domain. IEEE Transactions on Signal Processing, 49, 2545-2548. http://dx.doi.org/10.1109/78.960402

[10] Mustard, D. (1991) Uncertainty Principle Invariant under Fractional Fourier Transform. Journal of the Australian Mathematical Society (Series B), 33, 180-191. http://dx.doi.org/10.1017/S0334270000006986

[11] Stern, A. (2007) Sampling of Compact Signals in Offset Linear Canonical Transform Domains. Signal, Image and Video Processing, 1, 359-367. http://dx.doi.org/10.1007/s11760-007-0029-0

[12] Aytur, O. and Ozaktas, H.M. (1995) Non-Orthogonal Domains in Phase Space of Quantum Optics and Their Relation to Fractional Fourier Transforms. Optics Communications, 120, 166-170. http://dx.doi.org/10.1016/0030-4018(95)00452-E

[13] Stern, A. (2008) Uncertainty Principles in Linear Canonical Transform Domains and Some of Their Implications in Optics. Journal of the Optical Society of America A, 25, 647-652. http://dx.doi.org/10.1364/JOSAA.25.000647

[14] Sharma, K.K. and Joshi, S.D. (2008) Uncertainty Principle for Real Signals in the Linear Canonical Transform Domains. IEEE Transactions on Signal Processing, 56, 2677-2683. http://dx.doi.org/10.1109/TSP.2008.917384

[15] Zhao, J., Tao, R., Li, Y.L. and Wang, Y. (2009) Uncertainty Principles for Linear Canonical Transform. IEEE Transactions on Signal Processing, 57, 2856-2858. http://dx.doi.org/10.1109/TSP.2009.2020039

[16] Xu, G., Wang, X. and Xu, X. (2009) Three Cases of Uncertainty Principle for Real Signals in Linear Canonical Transform Domain. IET Signal Processing, 3, 85-92. http://dx.doi.org/10.1049/iet-spr:20080019

[17] Xu, G., Wang, X. and Xu, X. (2009) New Inequalities and Uncertainty Relations on Linear Canonical Transform Revisit. EURASIP Journal on Advances in Signal Processing, 2009, Article ID: 563265.

[18] Xu, G., Wang, X. and Xu, X. (2009) Generalized Entropic Uncertainty Principle on Fractional Fourier Transform. Signal Processing, 89, 2692-2697. http://dx.doi.org/10.1016/j.sigpro.2009.05.014

[19] Xu, G., Wang, X. and Xu, X. (2009) Uncertainty Inequalities for Linear Canonical Transform. IET Signal Processing, 3, 392-402. http://dx.doi.org/10.1049/iet-spr.2008.0102 
[20] Xu, G., Wang, X. and Xu, X. (2009) The Logarithmic, Heisenberg's and Short-Time Uncertainty Principles Associated with Fractional Fourier Transform. Signal Process, 89, 339-343. http://dx.doi.org/10.1016/j.sigpro.2008.09.002

[21] Xu, G., Wang, X. and Xu, X. (2010) On Uncertainty Principle for the Linear Canonical Transform of Complex Signals. IEEE Transactions on Signal Processing, 58, 4916-4918. http://dx.doi.org/10.1109/TSP.2010.2050201

[22] Xu, G., Wang, X. and Xu, X. (2010) Novel Uncertainty Relations in Fractional Fourier Transform Domain for Real Signals. Chinese Physics B, 19, Article ID: 014203. http://dx.doi.org/10.1088/1674-1056/19/1/014203

[23] Somaraju, R. and Hanlen, L.W. (2006) Uncertainty Principles for Signal Concentrations. Proceedings of 7th Australian Communications Theory Workshop, Perth, 1-3 February 2006, 38-42. http://dx.doi.org/10.1109/ausctw.2006.1625252

[24] Donoho, D.L. and Stark, P.B. (1989) Uncertainty Principles and Signal Recovery. SIAM Journal on Applied Mathematics, 49, 906-931. http://dx.doi.org/10.1137/0149053

[25] Donoho, D.L. and Huo, X. (2001) Uncertainty Principles and Ideal Atomic Decomposition. IEEE Transactions on Information Theory, 47, 2845-2862. http://dx.doi.org/10.1109/18.959265

[26] Elad, M. and Bruckstein, A.M. (2002) A Generalized Uncertainty Principle and Sparse Representation in Pairs of Bases. IEEE Transactions on Information Theory, 48, 2558-2567. http://dx.doi.org/10.1109/TIT.2002.801410

[27] Averbuch, A., Coifman, R.R., Donoho, D.L., Eladd, M. and Israeli, M. (2006) Fast and Accurate Polar Fourier Transform. Applied and Computational Harmonic Analysis, 21, 145-167. http://dx.doi.org/10.1016/j.acha.2005.11.003

[28] Pei, S.C. and Ding, J.J. (2007) Eigenfunctions of Fourier and Fractional Fourire Transforms with Complex Offsets and Parameters. IEEE Transactions on Circuits and Systems I: Regular Papers, 54, 1599-1611. http://dx.doi.org/10.1109/TCSI.2007.900182

[29] Pei, S.C., Yeh, M.H. and Luo, T.L. (1999) Fractional Fourier Series Expansion for Finite Signals and Dual Extension to Discrete-Time Fractional Fourier Transform. IEEE Transactions on Signal Processing, 47, 2883-2888. http://dx.doi.org/10.1109/78.790671

[30] Pei, S.C. and Ding, J.J. (2003) Eigenfunctions of the Offset Fourier, Fractional Fourier, and Linear Canonical Transforms. Journal of the Optical Society of America A, 20, 522-532. http://dx.doi.org/10.1364/JOSAA.20.000522

[31] Qi, L., Tao, R., Zhou, S. and Wang, Y. (2004) Detection and Parameter Estimation of Multicomponent LFM Signal Based on the Fractional Fourier Transform. Science in China Series F: Information Sciences, 47, 184-198. http://dx.doi.org/10.1360/02yf0456

[32] Pei, S.C. and Ding, J-J. (2000) Closed-Form Discrete Fractional and Affine Fourier Transforms. IEEE Transactions on Signal Processing, 48, 1338-1356. http://dx.doi.org/10.1109/78.839981

[33] Xia, X.G. (1996) On Bandlimited Signals with Fractional Fourier Transform. IEEE Signal Processing Letters, 3, $72-74$. http://dx.doi.org/10.1109/97.481159

[34] Tao, R., Li, Y.L. and Wang, Y. (2010) Short-Time Fractional Fourier Transform and Its Applications. IEEE Transactions on Signal Processing, 58, 2568-2580. http://dx.doi.org/10.1109/TSP.2009.2028095

[35] Tao, R., Deng, B., Zhang, W.Q. and Wang, Y. (2008) Sampling and Sampling Rate Conversion of Band Limited Signals in the Fractional Fourier Transform Domain. IEEE Transactions on Signal Processing, 56, 158-171. http://dx.doi.org/10.1109/TSP.2007.901666

[36] Wang, X., Xu, G., Ma, Y., Zhou, L. and Wang, L. (2013) Generalized Parseval's Theorem on Fractional Fourier Transform for Discrete Signals and Filtering of LFM Signals. Journal of Signal and Information Processing, 4, $274-$ 281. http://dx.doi.org/10.4236/jsip.2013.43035 